\title{
Modélisation numérique du comportement dynamique des joints annulaires d'étanchéité
}

\author{
Digital modelling of annular seals dynamic behaviour
}

\author{
par M. Arghir
}

Laboratoire de Mécaniques des Solides, Université de Poitiers

The work presents a method for analyzing the dynamic regime of labyrinth liquid seals. By using the traditional simplifying assumptions for the centered seal (sinusoidal, harmonically varying, first order pertubation), the approach can be addressed as 'quasi' $2 D$. A numerical coordinate transformation capable to treat displacement pertubations is introduced. The first order mathematical model is then deduced following the same steps as in previously published papers $[1,2]$. From this standpoint, the present method can be regarded as an extension of the above mentioned approaches that were able to deal only with statorgrooved seals. The method is validated by comparisons with Nordmann and Dietzen's [3] theoretical results for a seal with grooves on both stator and rotor and with the experimental results of Staubli's [4] test case concerning a general seal..

\section{IINTRODUCTION}

Pendant la dernière décennie, les méthodes théoriques pour l'analyse des joints annulaires sont devenues des outils élaborés et ont atteint une certaine maturité. Selon le modèle mathématique utilisé, on peut distinguer les méthodes basées sur l'hypothèse du film mince (fondement de la théorie de la lubrification quand les forces d'inertie sont importantes) et les méthodes basées sur les équations de Navier-Stokes complètes. Les méthodes appartenant à la première catégorie prédisent avec précision les caractéristiques d'une large classe de paliers, butées et joints annulaires lisses et sont utilisées avec plus ou moins de succès pour l'analyse des joints annulaires à stator rainuré. Les méthodes appartenant à la deuxième catégorie sont utilisées seulement quand il est nécessaire de dépasser des limitations de l'hypothèse du film mince. L'écoulement étant dominé par d'importantes zones de recirculation, l'analyse des joints labyrinthes est un réel problème.

L'analyse présentée appartient aux méthodes basées sur les équations de Navier-Stokes, et donc seulement les méthodes de cette classe seront brièvement discutées. Dans toutes les approches, la vibration est modélisée comme une précession constante du rotor sur une orbite circulaire. L'alternative serait l'intégration pas à pas du système nonlinéaire (rotor, paliers, joints, etc.) mais il apparaît que les forces et les moments obtenus avec le modèle simplifié sont assez précis pour l'analyse dynamique des lignes d'arbre. Après cette simplification, le problème peut être décrit comme la solution d'un écoulement non-stationnaire (mais avec une variation harmonique), turbulent et tridimensionnel
(3D). Les algorithmes numériques de la Mécanique des fluides sont assez matures pour permettre de telles solutions. Cette voie a été adoptée par quelques auteurs $[5,6,7]$ et ces approches n'ont pas a priori de limitations mais l'effort de calcul est assez important.

Une voie pour la réduction du temps de calcul est de supposer que l'amplitude de la vibration est petite par rapport à l'épaisseur du film. Suite à cette hypothèse traditionnelle, les variables de l'écoulement et les caractéristiques géométriques du joint sont exprimées en terme de petites perturbations autour de la position de fonctionnement centré. Les équations à l'ordre un qui en résultent sont linéaires mais fortement couplées. Les forces et les moments calculés pour plusieurs fréquences de précession servent pour obtenir les coefficients utilisés dans l'analyse dynamique des lignes d'arbre. Des modèles 3D à l'ordre un de ce type ont été présentés par Nordmann et Dietzen [5], Baskharone et Hensel [6], Athavale et Hendricks [7]. La résolution numérique nécessite moins de temps de calcul que l'approche non-perturbée, mais, en raison de la géométrie spécifique des labyrinthes, l'effort de calcul est toujours important.

Pour des positions de fonctionnement centré du joint, l'effort de calcul peut être réduit d'une manière importante en considérant que les variables de l'écoulement perturbé ont seulement une variation sinusoïdale dans la direction circonférentielle. Le problème à l'ordre zéro devient axisymétrique (2D) et en exprimant analytiquement $\partial / \partial \theta$, les équations à l'ordre un ont une forme similaire. En raison des trois composantes de la vitesse dans l'équation de continuité à l'ordre un, ces approches peuvent être caractérisées comme « quasi » 2D et le temps de calcul qu'elles nécessitent est évidemment moins important que dans les méthodes précé- 
dantes. La principale limitation est que toutes les approches proposées $[8,9,1,2]$ sont basées sur une transformation de coordonnées analytique qui limite l'application à des joints à stator rainuré et rotor lisse. Pour un utilisateur industriel, l'inconvénient peut être assez sévère si l'on prend en compte que pour ce type de joints annulaires il dispose aussi des méthodes simplifiées à trois volumes de contrôle $[10,11]$. Ces approches simplifiées ont d'autres problèmes (une forte dépendance des paramètres spécifiés par l'utilisateur) mais l'analyse des joints à stator rainuré et rotor lisse peut être effectuée avec un temps de calcul négligeable.

Le but du travail qui sera ensuite présenté est d'introduire une méthode "quasi » 2D pour l'analyse des joints labyrinthes de forme générale, fonctionnant avec des fluides incompressibles. La méthode peut être regardée comme une extension des approches déjà présentées $[1,2]$ qui sont incluses comme des cas particuliers. La méthode a été développée pour prendre en compte des vibrations cylindriques ou coniques mais il paraît que les vibrations de mésalignement n'ont jamais constitué un problème pour des joints labyrinthes de forme générale et les données nécessaires à la validation manquent complètement dans la littérature. On va donc présenter ensuite seulement la première partie de la méthode, traitant des vibrations de déplacement et ses validations.

\section{II — LE MODÈLE MATHÉMATIQUE}

Le fondement mathématique est constitué des équations de Navier-Stokes incompressibles, isothermes, 3D, exprimées dans des coordonnées cylindriques et associées au modèle de turbulence $k-\varepsilon$ (Launder and Spalding, 1974). Les conditions d'adhérence du fluide à la paroi et une loi logarithmique rugueuse [12] sont utilisées pour estimer les contraintes tangentielles à la paroi sur des maillages relativement grossiers. L'interaction du joint avec l'écoulement principal est décrite en imposant soit le débit de fuites dans le labyrinthe soit la différence de pression entre les chambres amont et aval, la vitesse circonférentielle et le niveau de turbulence dans la section d'entrée. Un écoulement avec des lignes de courant lisses, sans zones de recirculation, est imposé dans la section de sortie.

\subsection{L'expression de la transformation de coordonnées}

Le joint labyrinthe est décrit comme une succession de rectangles " élémentaires ». Par exemple, la géométrie du joint présenté sur la Figure 1a peut être décomposée en trois rectangles, $R_{1} R_{2} S_{2} S_{1} R_{1}, R_{2} R_{3} R_{4} R_{5} S_{5} S_{4} S_{3} S_{2} R_{2}$ et $R_{5} R_{6} S_{6} S_{5} R_{5}$. Pour des conditions de fonctionnement normales, le rotor est centré et le domaine compris entre le rotor et le stator est axi-symétrique. A cause du régime dynamique, le rotor est déplacé et les rectangles décrivant la géométrie du labyrinthe résultent modifiés (fig. $1 b$ ). La déformation des rectangles peut être décrite par une transformation de coordonnées entre le domaine axi-symétrique $\xi \eta \zeta$ et le domaine perturbé $x r \theta$ (les deux systèmes de coordonnées étant cylindriques). La position perturbée (ou l'excentricité) d'un point $P$ sur la frontière d'un rectangle "élémentaire " peut être exprimée comme (fig. $l b)$ :

$$
\begin{aligned}
& x(\xi, \eta, \varsigma, \tau)=\xi, \theta(\xi, \eta, \varsigma, \tau)=\zeta, t(\xi, \eta, \varsigma, \tau)=\tau \\
& r(\xi, \eta, \zeta, \tau)=\left\{\begin{array}{lrr}
\eta+e \hat{h}(\varsigma, \tau), & P \in \text { Rotor } \\
\eta+\frac{r_{\text {Stat }}-\eta}{C_{0}} e \hat{h}(\varsigma, \tau), & \text { Rotor }<P<\text { Stator } \\
\eta & , & P \in \text { Stator }
\end{array}\right.
\end{aligned}
$$

Les deux hypothèses simplificatrices utilisées traditionnellement pour l'analyse du régime dynamique sont introduites à l'aide de la fonction complexe $\hat{h}(\zeta, r)=\exp (-i \theta) \exp (i \Omega t)$. La première fonction exponentielle exprime le fait que la hauteur du rectangle élémentaire a une variation sinusoïdale dans la direction circonférentielle, signifiant que seule la première composante de Fourier a été prise en compte. La deuxième exponentielle est introduite afin de prendre en compte la précession du rotor dans la situation quand le système de coordonnée est fixé sur le stator. L'angle circonférentiel $\theta$ est mesuré comme sur la figure 2 . Ce modèle simplifié, plus ou moins traditionnel, est largement présenté dans la littérature (par exemple, Childs, 1993) [13].

L'amplitude de la précession est supposée petite par rapport à l'épaisseur du film. Le petit paramètre est défini par

\begin{tabular}{|c|c|c|c|}
\hline \multirow{7}{*}{$\begin{array}{l}\tilde{a}, \tilde{b}, \tilde{f}, \tilde{g}, \\
C, \\
e_{,}, \\
F_{X}, F_{r^{\prime}} \\
F_{Y}, F_{r}\end{array}$} & \multicolumn{2}{|c|}{ NOMENCLATURE } & \\
\hline & fonctions de la transformation numérique de & $\varepsilon$, & paramètre de perturbation \\
\hline & coordonnées & $\mu$, & viscosité dynamique, [Pa.s] \\
\hline & jeu, épaisseur du film, $[\mathrm{m}]$ & $\rho$, & densité, $\left[\mathrm{kg} / \mathrm{m}^{3}\right]$ \\
\hline & excentricité, $[\mathrm{m}]$ & $\sigma$ & coefficient de prérotation, $W / \omega r_{p}$ \\
\hline & force radiale, $[\mathrm{N}]$ & $\Omega$, & fréquence de précession, [ $\mathrm{rad} / \mathrm{sec}]$ \\
\hline & force tangentielle, $[\mathrm{N}]$ & $\omega_{3}$ & vitesse angulaire, $[\mathrm{rad} / \mathrm{sec}]$ \\
\hline in, jn, & volumes de contrôle dans la direction axiale & $\mid \vec{\omega}$ & module de la vorticité, [1/s] \\
\hline & et radiale & $\xi, \eta, \zeta$ & coordonnées transformées \\
\hline$k$, & énergie cinétique turbulente, $\left[\mathrm{m}^{2} / \mathrm{s}^{2}\right]$ & & coefficients de perte de charge \\
\hline$L$, & longueur du joint, $[\mathrm{m}]$ & $\Re$, & partie réelle \\
\hline$n$, & exposant de la loi « $1 / 7 »$ du profil des & Indices & \\
\hline$O X Y Z$, & système de référence fixe & in/ex, & section d'entrée/sortie \\
\hline & pression, $[\mathrm{Pa}]$ & $R / S$, Rot/Stat, & rotor/stator \\
\hline$R e$, & nombre de Reynolds & & turbulent \\
\hline$S_{\Phi}$, & terme source & $0 / 1$, & variables à l'ordre zéro/un \\
\hline & niveau de turbulence & ref, & valeur de référence \\
\hline$t, \tau$ & temps, [s] & Exposants & \\
\hline$u, v, w$, & composantes de la vitesse en coordonnées & $\hat{\imath}, \sim$ & valeur complexe \\
\hline$U, W, P$, & variables moyennées sur l'épaisseur du film & & $\begin{array}{l}\text { valeur sur la frontière du rectangle élémen- } \\
\text { taire }\end{array}$ \\
\hline $\begin{array}{l}x, r, \theta \\
y\end{array}$ & $\begin{array}{l}\text { coordonnées cylindriques } \\
\text { distance normale à la paroi, }[\mathrm{m}]\end{array}$ & 0 , & $\begin{array}{l}\text { valeurs totales (freinées) dans les chambres } \\
\text { amont et aval }\end{array}$ \\
\hline
\end{tabular}
rapport à la perturbation maximale admissible qui évite le 


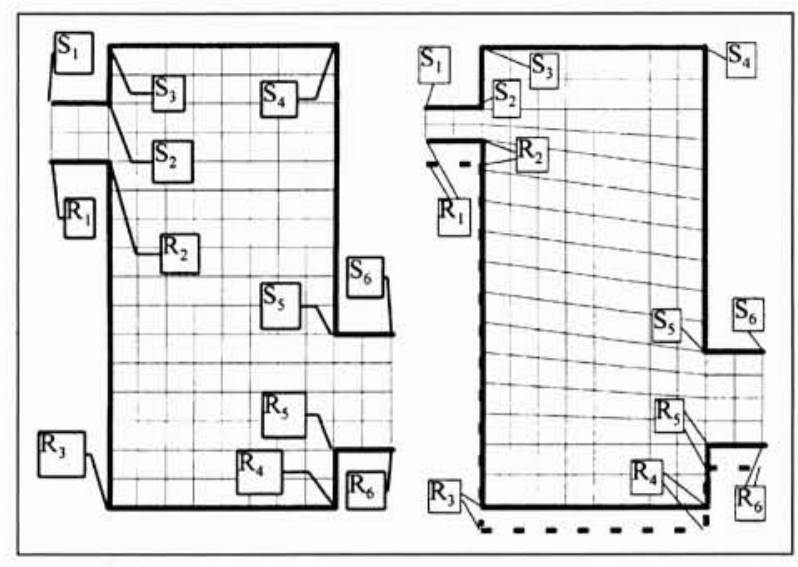

1. L'effet de la transformation de coordonnées (a. domaine axi-symétrique, b. perturbation de déplacement).

contact entre le rotor et le stator. Pour des vibrations de déplacement :

$$
\begin{aligned}
& C_{r e f}=e_{\text {max admiss. }}=\min \left\{C_{0}(x), 0 \leq x \leq L\right\} \\
& e=\frac{e}{C_{r e f}} C_{r e f}=\varepsilon \cdot C_{r e f} \quad \varepsilon=\frac{e}{C_{r e f}}
\end{aligned}
$$

Après l'introduction du paramètre de perturbation, la transformation décrivant le déplacement des frontières du rectangle " élémentaire » s'exprime :

$$
\begin{aligned}
& x(\xi, \eta, \zeta, \tau)=\xi, r(\xi, \eta, \zeta, \tau)=\eta+\varepsilon \tilde{f} *(\xi, \eta, \zeta, \tau), \theta(\xi, \eta, \zeta, \tau)= \\
& =\zeta, t(\zeta, \eta, \zeta, \tau)=\tau \\
& \tilde{f}^{*}(\xi, \eta, \zeta, \tau)= \begin{cases}C_{r e f} \hat{h}(\varsigma, \tau) & , P \in \text { Rotor } \\
\frac{r_{\text {Stat }}-\eta}{C_{0}} C_{r e f} \hat{h}(\zeta, \tau) & , \text { Rotor }<P<\text { Stator } \\
0 & , P \in \text { Stator }\end{cases}
\end{aligned}
$$

où désigne une variable contenant une partie réelle multipliée par $\hat{h}(\zeta, \tau)$.

La fonction $f^{*}$ est définie sur la frontière du rectangle élémentaire. La transformation de coordonnées pour les nœuds intérieurs est déduite en utilisant une relation d'interpolation linéaire entre les points situés sur la frontière droite (aval) et les points correspondants sur la frontière gauche (amont) de chaque rectangle. La correspondance est immédiate pour un maillage structuré.

$\tilde{f}(\xi, \eta, \zeta, \tau)=\left(\tilde{f}^{*}\right)_{\text {upstr. }}+\frac{\left(\tilde{f}^{*}\right)_{\text {downstr. }}-\left(\tilde{f}^{*}\right)_{\text {upstr. }}}{\xi_{\text {downstr. }}-\xi_{\text {upstr. }}}\left(\xi-\xi_{\text {upstr. }}\right)$

par exemple, dans le rectangle $R_{2} R_{3} R_{4} R_{5} S_{5}, S_{4} S_{3} S_{2} R_{2}$, $\left(\tilde{f}^{*}\right)_{\text {upstr. }} \equiv\left(\tilde{f}^{*}\right)_{R_{3} R_{2} R_{2} R_{3}}$ et $\left(\tilde{f}^{*}\right)_{\text {downstr. }} \equiv\left(\tilde{f}^{*}\right)_{R_{4} R_{5} R_{5} R_{4}}$.

\subsection{La matrice jacobienne de la transformation de cor- données}

La transformation de coordonnées introduite permet d'exprimer l'inverse de la matrice jacobienne nécessaire pour écrire les équations transformées. Tenant compte de l'effet de la linéarisation introduit par le petit paramètre, la matrice jacobienne peut être déduite analytiquement.

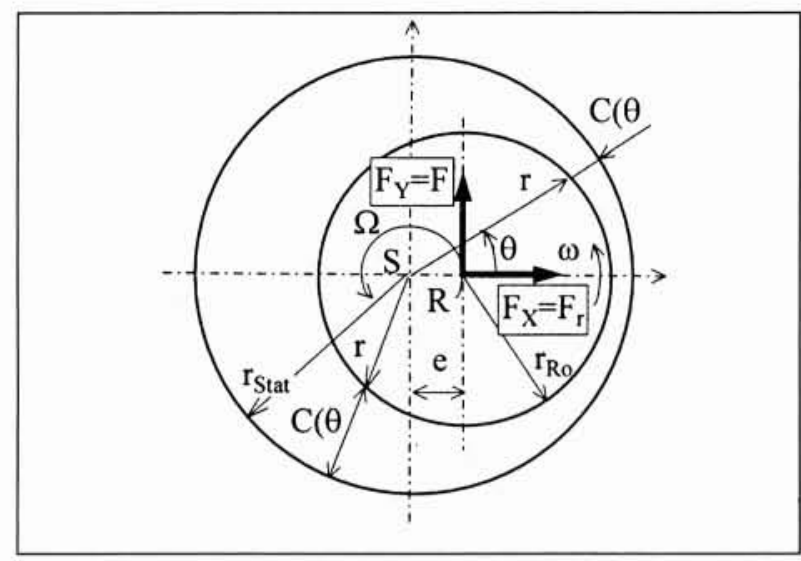

2. Forces sur le rotor en précession.

$$
\left[\begin{array}{l}
\partial / \partial x \\
\partial / \partial r \\
\partial / \partial \theta \\
\partial / \partial t
\end{array}\right]=\left[\begin{array}{rrrr}
1 & -\varepsilon \cdot \tilde{a} & 0 & 0 \\
0 & 1-\varepsilon \cdot \tilde{b} & 0 & 0 \\
0 & \varepsilon \cdot i \tilde{f} & 1 & 0 \\
0 & \varepsilon \cdot(-i \Omega \tilde{f}) & 0 & 1
\end{array}\right] \cdot\left[\begin{array}{l}
\partial / \partial \xi \\
\partial / \partial \eta \\
\partial / \partial \zeta \\
\partial / \partial \tau
\end{array}\right]
$$

où le déterminant est $1-\varepsilon \tilde{b}$ et

$$
\tilde{a}(\xi, \eta, \zeta, \tau)=\frac{\partial \tilde{f}}{\partial \xi}, \tilde{b}(\xi, \eta, \zeta, \tau)=\frac{\partial \tilde{f}}{\partial \eta}
$$

Les fonctions $\tilde{a}$, $\tilde{b}$, ont été introduites pour simplifier les notations. Les dérivées $\partial \widetilde{\Phi} / \partial \zeta=-i \widetilde{\Phi}, \partial \widetilde{\Phi} / \partial \tau=i \Omega \widetilde{\Phi}, \Phi \in$ $\{\tilde{f}, \tilde{a}, \tilde{b}\}$ sont exprimées analytiquement, mais les valeurs dans les nœuds du maillage et les dérivées $\partial / \partial \xi$, $\partial / \partial \eta$ sont estimées numériquement à l'aide des différences finies centrées.

\subsection{Les équations à l'ordre un}

Afin d'obtenir le modèle mathématique à l'ordre un, les variables de l'écoulement sont exprimées en utilisant les hypothèses simplificatrices citées avant (variation sinusoïdale, harmonique, perturbation à l'ordre un).

$$
\begin{aligned}
& \Phi(\xi, \eta, \zeta, \tau)=\Phi_{0}(\xi, \eta)+\varepsilon \Phi_{1}(\xi, \eta, \zeta, \tau)+\varepsilon^{2} \ldots, \Phi_{1}(\xi, \eta, \zeta, \tau) \\
& =\Re\left[\Phi_{1}(\xi, \eta) \hat{h}(\zeta, \tau)\right], \Phi_{1}=\Phi_{1 \cos }+i \Phi_{1_{\text {sin }}}
\end{aligned}
$$

où $\Phi \in\{u, v, w, p, u\}$. Ces relations et la transformation de coordonnées (7) sont systématiquement introduites dans les équations 3D et dans les conditions aux limites.

Les équations à l'ordre zéro décrivent l'écoulement axisymétrique (2D) stationnaire et leurs expressions ont été présentées ailleurs [2]. Les équations à l'ordre un sont $3 \mathrm{D}$ et non-stationnaires. L'hypothèse d'une variation sinusoïdale des variables de l'écoulement permet d'écrire les équations à l'ordre un sous la même forme que les équations à l'ordre zéro. Pour faciliter l'intégration numérique, elles sont exprimées comme des équations générales de transport 2D.

$$
\frac{\partial}{\partial \xi}\left(\rho u_{0} \hat{\Phi}_{1}\right)+\frac{1}{\eta} \frac{\partial}{\partial \eta}\left(\eta \rho v_{0} \hat{\Phi}_{1}\right)=\frac{\partial}{\partial \xi}\left(\mu_{0} \frac{\partial \hat{\Phi}_{1}}{\partial \xi}\right)+\frac{1}{\eta} \frac{\partial}{\partial \eta}\left(\eta \mu_{0} \frac{\partial \hat{\Phi}_{1}}{\partial \eta}\right)+\hat{S}_{\Phi}
$$

où $\Phi \in\{\hat{u}, \hat{v}, \hat{w}\}$. L'équation de continuité à l'ordre un s'écrit : 
$\frac{\partial}{\partial \xi}\left(\rho \hat{u}_{1}\right)+\frac{1}{\eta} \frac{\partial}{\partial \eta}\left(\eta \rho \hat{v}_{1}\right)=i \frac{\rho}{\eta} \hat{w}_{1}+\left[\tilde{a} \frac{\partial\left(\rho u_{0}\right)}{\partial \eta}+\tilde{b} \frac{\partial\left(\rho v_{0}\right)}{\partial \eta}+\dot{\xi}\right.$

$\left.+\tilde{g} \frac{\rho v_{0}}{\eta}-i \tilde{g} \frac{\partial\left(\rho w_{0}\right)}{\partial \eta}\right]$

Comme pour les modèles antérieurs $[1,2]$. l'effet de la perturbation de la viscosité turbulente est pris en compte.

\subsection{Conditions aux limites}

Les conditions d'adhérence du fluide à la paroi s'écrivent :

Stator: $\hat{u}_{1}=(0,0), \hat{v}_{1}=(0,0), \hat{w}_{1}=(0,0)$,

Rotor : $\hat{u}_{1}=(0,0), \hat{\mathrm{v}}_{1}=\left[0,(\Omega-\omega) C_{r e f}\right], \hat{w}_{1}=\left[\Omega C_{r e f} 0\right]$

Pour l'écoulement turbulent, les conditions aux limites à l'ordre un sont obtenues par la perturbation du profil universel de vitesses. Comme pour l'écoulement à l'ordre zéro, ces conditions permettent l'utilisation des maillages grossiers près des parois.

Les conditions à l'entrée et à la sortie s'écrivent :

$$
\begin{aligned}
& \left(\hat{U}_{i n}\right)_{1}=-\frac{\left(\hat{P}_{i n}\right)_{1}}{\rho\left(U_{i n}\right)_{0}\left(1+\xi_{i n}\right)},\left(\hat{v}_{i n}\right)_{1}=\left(\hat{w}_{i n}\right)_{1}=0, \\
& \left(\hat{P}_{e x}\right)_{1}=\left(\xi_{e x}-1\right) \rho\left(U_{e x}\right)_{0}\left(\hat{U}_{e x}\right)_{1}, \frac{\partial^{2} \hat{\Phi}_{1}}{\partial x^{2}}=0, \hat{\Phi}_{1}=\left\{\hat{u}_{1}, \hat{v}_{1}, \hat{w}_{1}\right\}
\end{aligned}
$$

Les conditions de type gradient, le traitement spécial de $\hat{v}_{1}$ imposé par la présence des termes sources dans l'équation de continuité ainsi que l'inclusion de l'effet de récupération de pression dans la section de sortie sont d'autres points importants du modèle mathématique. Le traitement est similaire au cas de la transformation analytique de coordonnées $[1,2]$ et les relations peuvent être déduites facilement.

\section{III — RÉSULTATS ET DISCUSSIONS}

Les équations sont discrétisées en utilisant la méthode des volumes finis et un maillage structuré à volumes de contrôle décalés pour les composantes axiales et radiales de la vitesse. Des résultats pour deux joints labyrinthes de forme générale sont ensuite présentés.

\section{- 3.1 Comparaisons avec les résultats de Nordmann et Dietzen [3]}

Un des premiers résultats théoriques a été obtenu par Nordmann et Dietzen [3] pour la géométrie présentée sur la figure $3\left(\rho=996 \mathrm{~kg} / \mathrm{m}^{3}, \mu=0,7.10^{-3} \mathrm{~Pa} . \mathrm{s}\right.$, différence de pression imposée $\Delta P^{0}=0,8 \mathrm{MPa}, \xi_{\text {in }}=0,5, \xi_{\text {ex }}=1, \omega=418,88 \mathrm{rad} / \mathrm{s}$, $\sigma=0,5$ et parois hydrauliques lisses). Les auteurs ont effectué un calcul 3D pour une excentricité $e=C_{0} / 40$. Les résultats de l'analyse présentée ont été obtenus pour un maillage avec 234 intervalles à pas variable dans la direction axiale et 22 intervalles à pas constant dans la direction radiale. La contribution de chaque zone du film et de chaque rainure est présentée sur la figure 4. La valeur totale de la raideur directe est en bonne concordance avec les résultats de Nordmann et Dietzen mais la contribution de chaque zone est quelque peu différente. Dans les résultats de Dietzen, la contribution de chaque zone de film à la raideur directe décroît le long du joint, tandis que dans les calculs présentés leur apport est presque constant. Les différences sur la rai-

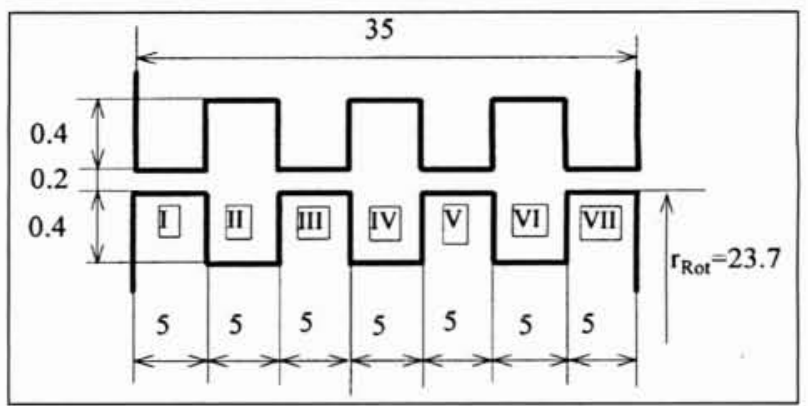

3. La géométrie du cas test de Nordmann et Dietzen [3] (toutes les dimensions sont en $\mathrm{mm}$ ).

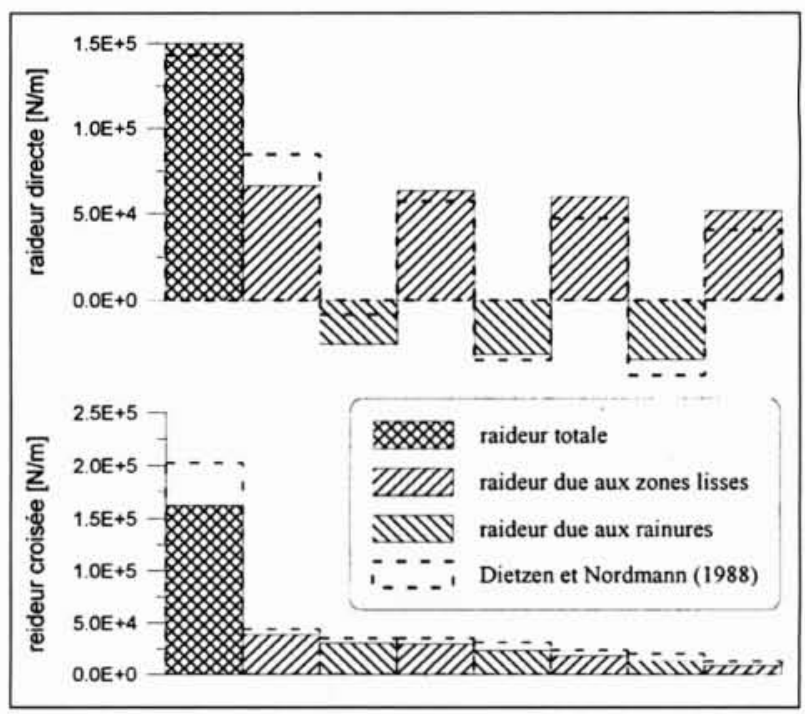

4. Raideurs directes et croisées.

deur croisée sont plus importantes mais la contribution de chaque zone a la même tendance que les résultats de Dietzen. Finalement, il est difficile de se prononcer pour un des résultats en l'absence de mesures ou d'un troisième résultat théorique.

\subsection{Résultats obtenus pour le cas test de Staubli [4]}

Un cas test basé sur des résultats expérimentaux a été proposé par Staubli [4]. La géométrie du joint est présentée sur la figure $5\left(\rho=998,5 \mathrm{~kg} / \mathrm{m}^{3}, \mu=10^{-3}\right.$ Pa.s, débit imposé $R e_{a x 1} / 2=14838, \xi_{\text {in }}=0,5, \xi_{e x}=1, \omega=104,72 \mathrm{rad} / \mathrm{s}, \sigma=$ $0,16(3 \mathrm{~m} / \mathrm{s}), n=7, T=2 \%$ et parois hydrauliques lisses). Les deux maillages utilisent le même nombre des volumes de contrôle dans les joints lisses (in $=16, j n=8$ dans le premier joint et in $=64, j n=8$ dans le deuxième joint) mais des maillages différents dans la chambre (in $=24, j n=48$ pour le premier maillage et in $=40, j n=72$ pour le deuxième maillage).

Les pressions à l'ordre zéro sur le stator sont présentées sur la figure 6. Les valeurs dans le deuxième joint sont en bonne concordance avec les mesures. Dans le premier joint, la dérivée des pressions est bien prédite mais les pressions absolues sont déplacées vers des valeurs plus importantes. Il résulte que l'écoulement dans la chambre agit comme une résistance hydraulique supplémentaire. Cette surestimation, qui ne peut pas être éliminée par des affinages raisonnables du maillage, est due au modèle de turbulence. Les pressions 


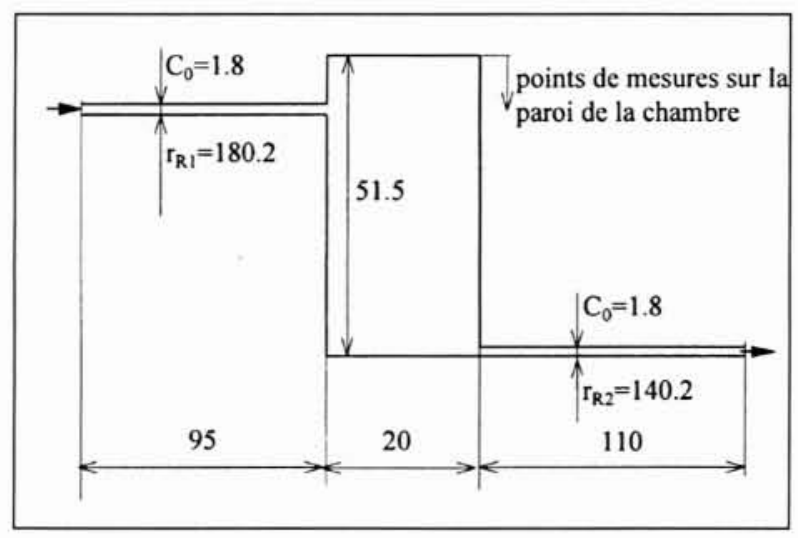

5. La géométrie du cas test de Staubli [4] (toutes les dimensions sont en mm).

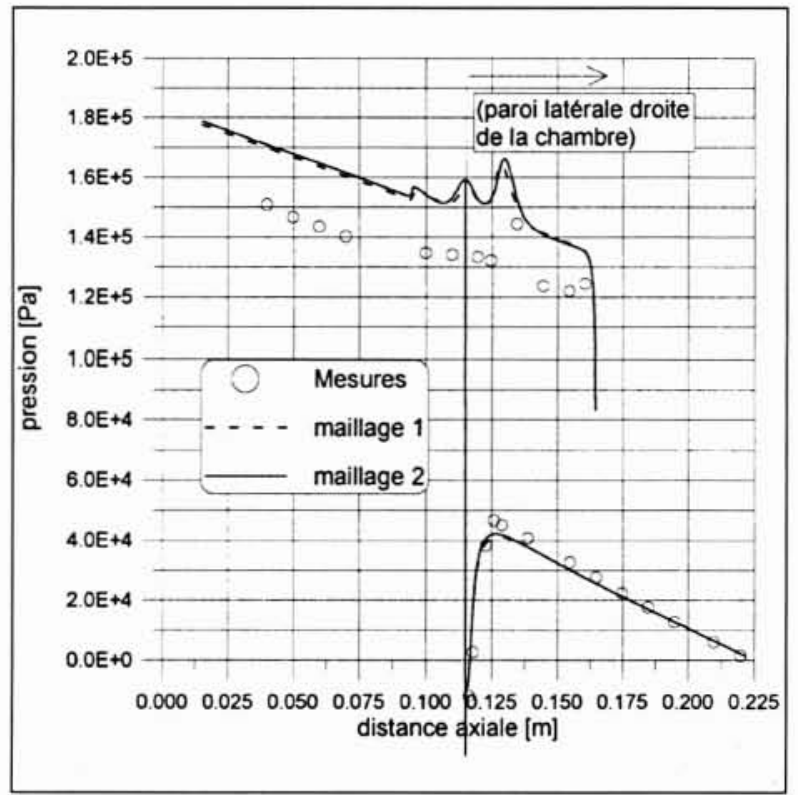

6. Pressions à l'ordre zéro sur le stator (fonctionnement centré).

expérimentales à l'ordre un (obtenues à partir de mesures effectuées pour une excentricité de $40 \%$ et précession nulle) sont comparées avec les résultats de calculs sur les figures 7 et 8 . Pour précession nulle, les composantes en cosinus et en sinus donnent la raideur directe et la raideur croisée. Il a été apprécié que cette comparaison caractérise mieux le champ de pression à l'ordre un que la comparaison avec le champ de pressions excentrées qui est normalement mesuré. Les résultats montrent une bonne concordance avec les mesures surtout pour la zone d'entrée du deuxième joint où tous les détails sont bien reproduits.

\section{IV $\square$ CONCLUSIONS}

La méthode numérique présentée permet l'analyse des joints labyrinthes de forme générale fonctionnant en position centrée. La méthode est basée sur les équations complètes de l'écoulement, et le point central de l'approche est une transformation numérique de coordonnées. Les hypothèses sim-



7. Pressions à l'ordre un sur le stator.

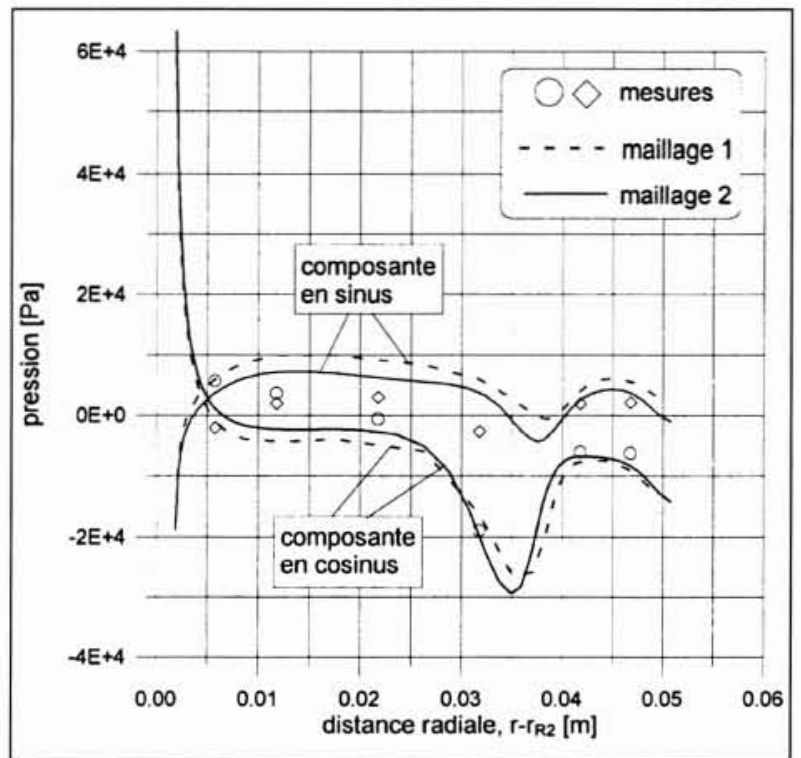

8. Pressions à l'ordre un sur la paroi latérale de la chambre.

plificatrices traditionnelles (petites perturbations, variation sinusoïdale et harmonique) rendent l'analyse « quasi » $2 \mathrm{D}$ et moins difficile que dans le cas des méthodes 3D. Il est à souligner que les méthodes « quasi 2D " présentées jusqu'à présent dans la littérature ne pouvaient être utilisées que pour l'analyse des joints annulaires à stator rainuré et rotor lisse. Finalement, les champs de pression obtenus pour deux joints de forme générale montrent une bonne concordance avec d'autres résultats théoriques ou expérimentaux.

\section{BIBLIOGRAPHIE}

[1] ARghir M., Frene J. (1997a), - « Forces And Moments Due to Misalignement Vibrations in Annular Liquid Seals Using The Averaged Navier-Stokes Equations ». ASME Journal of Tribology, Vol. 119, pp. 279-290. 
[2] ARghiR M., Frene J. (1997b). - « Rotordynamic Coefficients of Circumferentially-Grooved Liquid Seals Using the Averaged Navier-Stokes Equations ». ASME Journal of Tribology, Vol. 119, pp. 556-567.

[3] Nordmann R., Dietzen F.J. (1988). - « Finite Difference Analysis of Rotordynamic Seal Coefficients For An Eccentric Shaft Position ", presented at the Rotordynamics Instability Problems in High Performance Turbomachinery, proceedings of a workshop held at Texas A\&M University, 269-284.

[4] Staubli T. (1993). - «A Labyrinth Seal - ETHZ Test Case », presented at Société Hydrotechnique de France, October, Paris.

[5] Tam L.T., PrZekwas A.J., Muzsynska A., Hendricks R.C., Braun M.J., Mullen R.L. (1988). - " Numerical and Analytical Study of Fluid Dynamic Forces in Seals and Bearings ". ASME Journal of Vibration, Acoustics, Stress and Reliability in Design, Vol. 110, pp. 315-325.

[6] Baskharone E.A., Hensel S.J. (1991). - « A Finite-Element Perturbation Approach to Fluid/Rotor Interaction in Turbomachinery Elements. Part 1: Theory ». ASME Journal of Fluids Engineering, September, Vol. 113, pp. 353-361.

[7] Athavale M.M., Hendricks R.C. (1994), - « A Small Perturbation CFD Method for Calculation of Seal Rotordynamic Coefficients ", ISROMAC-5, Maui, Hawaii, May.

[8] Dietzen F.J., NoRdmann R. (1987). - « Calculating Rotordynamic Coefficients of Seals by Finite-Difference Techniques". ASME Journal of Tribology, Vol, 109, pp. 388-394.
[9] Feng T., Nordmann R. (1992). - « Identification of Fluid/Structure Interactionsin Centrifugal Pumps (part 1: Computational Procedure) », ISROMAC-4, Vol. A, Hawaii, USA, pp. 34-43.

[10] Florjancic S. (1990). — «Annular Seals of High Energy Centrifugal Pumps: A New Theory and Full Scale Measurement of Rotordynamic Coefficients and Hydraulic Friction Factors », Diss ETH Nr. 9087, Swiss Federal Institute of Technology, Zurich, Switzerland.

[11] Marquette O.R., Childs D.W. (1995). — « An Extended Three-Control-Volume Theory for Circumferentially-Grooved Liquid Seals ». ASME/STLE Tribology Conference, October 8-11, Orlando, FL, 95-TRIB-15.

[12] Кон Y.-M. (1992). — « Turbulent Flow Near a Rough Wall ». ASME Journal of Fluids Engineering. Vol. 114, pp. 537-542.

[13] CHILDS D.W. (1993). - Turbomachinery Rotordynamics : Phenomena, Modeling and Analysis, Wiley\&Sons, New York.

[14] Athavale M.M., Przekwas A.J., Hendricks R.C., Liang A. (1994), - «SCISEAL: A 3D CFD Code for Accurate Analysis of Fluid Flow and Forces in Seals ", Advanced ETO Propulsion Conference, May.

[15] Rhode D.L., Hensel S.J., Guidry M.J. (1992). — « Labyrinth Seal Rotordynamic Forces Using a Three-Dimensional Navier-Stokes Code ». ASME Journal of Tribology, Vol. 114, pp. 683-689. 